\title{
Problématique actuelle de l'irrigation en France
}

\author{
A. Roux, Ministère de l'Agriculture et de la Forêt et \\ Y. Mérillon, Ministère de l'Environnement
}

\section{Introduction}

Les années de sécheresse successives que vient de connaître la France en 1989, 1990 et 1991, l'accroissement rapide des stocks de céréales au niveau communautaire et la montée en puissance des préoccupations d'environnement ont placé l'irrigation au centre d'un débat social particulièrement agité.

En effet l'irrigation assimilée de manière simple à l'intensification fait l'objet de critiques acerbes de la part des milieux écologiques et on ne compte plus les colloques, séminaires et assises où cette technique est placée au banc des accusés. Pour certains la détérioration des milieux aquatiques due aux prélèvements d'eau excessifs, à la pollution par les engrais liée à l'intensification elle-même permise par l'irrigation sont à mettre au passif d'un bilan particulièrement catastrophique. Pour d'autres, les agriculteurs en particulier, cette technique ne présente pas que des inconvénients et ils continuent à en réclamer le développement au motif de la régularité des rendements, la qualité des produits et des possibilités de diversification, trois arguments auxquels les élus locaux ajoutent celui de l'aménagement du territoire et du maintien de la vie rurale dans des zones en difficultés économiques.

Le présent exposé n'a pas pour but de trancher dans ce débat mais de lui permettre de se développer dans un climat plus serein en présentant l'état des lieux, et les facteurs de changement du contexte dans lequel se situe l'irrigation aujourd'hui, en résumé de poser les éléments de la problématique actuelle de l'irrigation en France.

\section{Etat des lieux}

Depuis une vingtaine d'années les superficies agricoles irriguées ont plus que doublé passant de 600000 ha à 1200000 ha. Dans les années 80, l'accroissement annuel a atteint le niveau record de 40000 ha.

Ce développement s'il peut paraître important en valeur relative ne doit toutefois pas faire oublier que la part de la Surface Agricole Utile (SAU) du territoire français qui est irriguée chaque année n'est que de $4 \%$. A titre de comparaison nos principaux concurrents européens pour les cultures irriguées ont des taux bien supérieurs: Espagne : $12 \%$, Italie : $21 \%$, Grèce : $21 \%$ et Portugal : $9 \%$.
Une fois ces chiffres globaux rappelés, il convient de poursuivre l'analyse en détaillant ces données à partir de différentes approches.

\subsection{Répartition spatiale}

On remarque une répartition très inégale entre les différentes régions qui s'explique aisément par des raisons climatiques. L'annexe I montre l'importance des superficies irriguées et le pourcentage qu'elles représentent par rapport à la SAU régionale. On constate que si on ajoute aux régions qui dépassent la moyenne de $4 \%$ les surfaces irriguées des Pays de la Loire, on rassemble $93 \%$ de la superficie irriguée métropolitaine, déterminant ainsi un partage du territoire national entre régions très concernées par l'irrigation et les autres.

Si maintenant on s'intéresse à l'évolution constatée dans les vingt dernières années, on peut décomposer ces régions très concernées par l'irrigation en deux sous-ensembles :

- dans un premier groupe des régions méditerranéennes (Languedoc-Roussillon, Corse, Provence-Alpes-Côte d'Azur), la surface irriguée est restée stationnaire (autour de 200000 ha). Il s'agit dans cette zone d'une technique très ancienne où les accroissements constatés en Languedoc-Roussillon sont compensés par les abandons d'équipements peu rentables ;

- dans l'autre groupe par contre, les accroissements sont spectaculaires comme le montre le tableau figurant en annexe $I$.

\subsection{Répartition par cultures}

L'annexe II montre l'évolution des cultures irriguées entre 1975 et 1988.

La croissance relative la plus forte est à mettre au crédit des oléoprotéagineux, qui sont passés d'une surface quasinulle à 85000 ha. Mais c'est le maïs qui retient principalement l'attention : pendant cette même période, la superficie en maïs irriguée a plus que doublé et on peut dire qu'aujourd'hui, la moitié pratiquement de la sole irriguée métropolitaine lui est consacrée. La surface totale cultivée en maïs étant, pendant la même période, restée relativement constante (1900 000 ha environ), ce phénomène s'est accompagné d'un déplacement de cette culture vers le Centre-Ouest, le Sud-Ouest et l'Alsace. 


\subsection{Les exploitations et le mode d'irrigation}

136000 exploitations sont équipées pour l'irrigation pour 1797000 ha et 121000 irriguent effectivement chaque année 1147000 ha.

$\mathrm{Si}$ on s'intéresse aux régions les plus concernées telles que définies plus haut en fonction du taux de superficie irriguée, on remarque qu'elles ont toutes plus de $10 \%$ d'exploitations équipées pour l'irrigation : le pourcentage atteint $56 \%$ en Provence-Alpes-Côte d'Azur et dépasse $20 \%$ en Corse, Languedoc-Roussillon, Aquitaine et MidiPyrénées.

Une répartition des modes d'irrigation en fonction de la surface affectée à chacun d'entre eux montre une nette domination de l'aspersion.

$\begin{array}{ll}\text { Aspersion } & : 800000 \text { ha } \\ \text { Micro-irrigation } & : 17000 \text { ha } \\ \text { Gravité } & : 85000 \text { ha } \\ \text { Plusieurs systèmes } & : 245000 \text { ha }\end{array}$

Près d'une exploitation équipée sur deux est raccordée à un réseau collectif et près de deux sur trois disposent d'une ressource individuelle : certaines exploitations disposent donc d'une double ressource en eau.

Les ressources individuelles assurent l'alimentation en eau de 700000 ha.

Les consommations en eau par hectare varient largement en fonction des conditions climatiques locales, de la culture pratiquée et du mode d'irrigation. Si des chiffres exceptionnels de plus de $10000 \mathrm{~m}^{3}$ sont rencontrés dans les régions d'irrigation ancienne, le volume moyen consommé s'établit à $1800 \mathrm{~m}^{3} /$ ha.

\subsection{Les investissements}

Au cours de la période 1981-1988, le montant total des investissements d'irrigation, réalisé avec une aide publique, s'est élevé à 9 milliards de francs dont $90 \%$ a été réalisé dans les régions les plus concernées et s'est réparti de manière à peu près égale entre les régions méditerranéennes et les autres. Le taux de subvention moyen s'est établi à $56,5 \%$.

Il s'agit essentiellement de réseaux collectifs nécessitant des investissements de départ importants pour la mobilisation et le transport de l'eau.

Les différents partenaires impliqués dans le financement des équipements ont maintenu leur effort global mais la part relative de chacun a varié : l'aide de l'Etat a diminué de $45 \%$ à $22 \%$, tandis que celle des Régions a progressé de 6 à $9 \%$, celle des départements de 2 à $14 \%$ et celle des Agences de l'Eau de 3 à $13 \%$.

Il convient de noter également que $60 \%$ des surfaces irrigables représentant $40 \%$ des investissements l'a été sans subvention. Cette proportion atteint même $75 \%$ à $95 \%$ en Alsace, dans les Pays de la Loire, le Centre et le Poitou-Charentes. Ce type d'investissement concerne souvent des surfaces plus réduites et des systèmes intéressant une exploitation à partir d'une ressource facilement mobilisable (rivière ou forage).
Cet état de fait n'est probablement pas sans rapport avec la constatation que la gestion de la pénurie et de la crise des trois dernières années a été beaucoup plus délicate dans les trois régions précitées que plus au sud, dans le bassin de la Garonne où l'expérience de la gestion collective a bien facilité les choses.

\section{Les facteurs de changement}

Le développement important que l'irrigation a connu pendant ces vingt dernières années s'explique par la conjonction d'un certain nombre de facteurs favorables essentiellement économiques: la politique de soutien des prix conduite par la Politique Agriculture Commune, l'existence de taux d'intérêts quasi négatifs en raison de l'inflation et les taux de subvention importants accordés par les pouvoirs publics permettaient à l'agriculteur de se lancer dans ce type d'investissement sans courir de risques majeurs.

La situation n'est plus la même aujourd'hui et le contexte dans lequel le futur irrigant doit prendre sa décision n'est plus aussi favorable. Les paramètres extérieurs qui ont changé concernent les nouvelles données en matière de rémunération des agriculteurs, les préoccupations grandissantes de protection de l'environnement et des ressources naturelles, et les dispositions de la nouvelle loi sur l'eau.

\subsection{Les dispositions de la nouvelle Politique Agricole Commune}

L'examen des règlements pris en application des décisions arrêtées par les Ministres de l'Agriculture de la Communauté en juin 1992 montre qu'un certain nombre de dispositions ont une influence directe sur l'irrigation.

Tout d'abord il faut remarquer que la réforme de la PAC ne concerne qu'un certain nombre de cultures irriguées. L'examen des statistiques figurant en annexe $I I$ montre que $60 \%$ seulement des surfaces arrosées chaque année porte des cultures dont les règles de fonctionnement du marché seront modifiées : les cultures industrielles et les cultures spéciales, fruits et légumes en particulier, continueront à être soumises au même régime que par le passé et à subir la concurrence des autres pays producteurs européens.

Le règlement grandes cultures (céréales, oléagineux, protéagineux) prévoit la possibilité de définir au niveau départemental une surface plafond pour les cultures irriguées égale à la moyenne des années 1989, 1990 et 1991 à laquelle s'ajouteront les surfaces nouvellement irriguées à partir d'investissements déjà mis en œuvre le $1^{\mathrm{er}}$ août 1992. Cette mesure est particulièrement importante pour deux raisons. Tout d'abord en déterminant un paiement compensatoire majoré pour les irrigants actuels, elle sauvegarde l'équilibre financier des structures collectives (ASA ou Syndicats d'irrigation) dont la «faillite " aurait certainement eu un impact très négatif sur l'investissement futur dans ce domaine. Ensuite elle va conduire les candidats 
irrigants à viser plutôt des cultures hors PAC car il est douteux que le coût supplémentaire de l'hectare irrigué soit compensé par l'augmentation de revenu espérée avec des prix dont la décroissance est programmée et un montant compensatoire défini par hectare en augmentation mais tenant compte d'un rendement en sec.

Il faut donc s'attendre à une redéfinition des stratégies de la part des agriculteurs qui vont repenser leur système d'exploitation dans le but de maximiser leur revenu en faisant appel aux possibilités offertes par la nouvelle PAC incluant la mise en œuvre des mesures d'accompagnement dont la teneur n'est pas encore définitivement arrêtée. La décision de s'équiper pour l'irrigation sera prise dans ce contexte et dépendra également de la politique d'aide suivie par les pouvoirs publics dans le domaine de l'hydraulique agricole.

\subsection{Les préoccupations d'environnement}

Depuis plusieurs années déjà, partant de la constatation d'une dégradation continue de la qualité des milieux aquatiques malgré les efforts faits par les collectivités et les industriels, l'irrigation fait l'objet de vives critiques de la part des milieux écologiques.

Force est de constater que le développement de l'irrigation s'est accompagné d'un prélèvement d'eau augmentant dans des proportions quasi identiques. Si l'on raisonne en terme de consommation nette, c'est-à-dire de quantité soustraite au milieu naturel sans restitution plus ou moins rapprochée dans le temps ou dans l'espace, l'irrigation représente $44 \%$ du total, loin devant les autres utilisateurs. Si l'on ajoute à cette constatation le fait que la demande d'irrigation se situe pendant les périodes d'étiage que la création de ressources n'a pas suivi l'évolution des besoins, et que la France vient de connaître trois années de sécheresse consécutives, on comprend que le débat social soit parfois très vif.

L'une des raisons essentielles de réformer la PAC réside dans l'existence d'excédents céréaliers en constante augmentation ( 25 millions de tonnes au 31 décembre 1991) entraînant un accroissement corrélatif des dépenses de la section garantie du FEOGA. L'importance de ce surplus doit d'ailleurs être relativisé car l'Europe continue à importer du maïs (qui entre en Espagne) et des produits de substitution des céréales en vertu d'accords anciens. L'assimilation faite entre irrigation et intensification ne va pas manquer de relancer le débat social. Les agriculteurs doivent se préparer à ces discussions en approfondissant les études préalables à tout projet dans le sens d'une meilleure appréciation de l'impact environnemental mais aussi des conséquences micro (niveau de l'exploitation) et macro-économiques (niveau de la petite région). Ils doivent être en mesure de justifier auprès du corps social, constitué à $90 \%$ d'urbains, le bien-fondé de leurs besoins en comparaison des atteintes supportées par le milieu naturel, ainsi que de la charge financière supportée par l'Etat et les collectivités locales.

\subsection{La nouvelle loi sur l'eau}

Votée le 3 janvier 1992, une nouvelle loi sur l'eau complétant et remplaçant celle de 1964 fixe le cadre dans lequel les prélèvements d'eau peuvent être effectués. Même si tous les décrets correspondants ne sont pas encore pris, la loi elle-même par le nouvel élan qu'elle donne à la protection des milieux aquatiques et les dispositions qu'elle contient en matière de gestion de l'eau donne une bonne idée du paysage administratif dans lequel les irrigants vont devoir s'intégrer.

Même si les irrigants doivent s'attendre à une réglementation renforcée en matière d'autorisations de prélèvement, ce n'est pas à ce niveau que la nouvelle loi va définir une règle du jeu modifiée. C'est plutôt l'application de l'article 2 qui relève substantiellement la protection du milieu aquatique dans la hiérarchie des objectifs de la gestion de l'eau (le principe d'équilibre à respecter entre deux légitimités - la protection des milieux et la valorisation économique de l'eau - est affiché clairement) et celle de l'article 5 qui prévoit l'élaboration de Schémas d'Aménagement et de Gestion des Eaux qui auront une influence forte sur l'évolution de l'irrigation.

En effet au niveau d'un sous-bassin ou d'un groupe de sous-bassin donc d'une entité hydrographique homogène, une Commission Locale de l'Eau devra élaborer un schéma. Constituée pour moitié d'élus locaux, d'un quart de représentants des administrations et d'un quart de socioprofessionnels et d'associations, elle sera présidée par un élu et l'approbation du schéma devra être faite à la majorité des deux tiers.

Les agriculteurs par le biais de leurs organes représentatifs auront un rôle majeur à jouer dans la préparation de ces documents. C'est en effet à ce niveau que seront définies les quantités d'eau allouées à l'irrigation ainsi que le mode de répartition du rationnement en situation de pénurie.

Les irrigants actuels et potentiels devront donc participer aux travaux préparatoires à ces schémas en apportant le maximum d'études solidement argumentées justifiant leur demande auprès du corps social. Les analyses mentionnées au paragraphe précédent prennent donc toute leur valeur. Ils devront également démontrer toute l'attention qu'ils portent aux économies d'eau, dans leur intérêt bien compris d'ailleurs, puisqu'une fois les plafonds de consommations fixés par le SAGE, toute diminution des volumes unitaires nécessaires entraînera une augmentation des surfaces irrigables. Enfin les progrès importants accomplis sous la pression des sécheresses récentes, en matière d'organisation et de gestion des périodes de pénurie trouveront dans ces schémas l'occasion d'une application institutionnelle engageant les différents partenaires.

Les irrigants devront donc dans le futur apprendre à mieux utiliser la ressource en eau d'une part dans la limite globale qui leur aura été attribuée mais également à gérer des quantités limitées selon des scénarios de rationnement prévus à l'avance et mis en application en fonction des circonstances climatiques et hydrologiques. 


\section{Conclusion}

Conclure un exposé consacré à la définition d'une problématique est un exercice difficile. La véritable conclusion va être apportée par les orateurs qui vont suivre et qui vont traiter du futur de l'irrigation dans le contexte nouveau qui s'applique maintenant à l'agriculture française.

Néanmoins il convient de mettre en garde les différents partenaires impliqués sur les risques de dérive du débat qui va s'engager. Certes comme le montrent les éléments de problématique exposés dans cette présentation, le contexte dans lequel évolue l'irrigation a changé, mais pas au point de réduire le débat à une discussion de type idéologique global : faut-il ou non arrêter l'irrigation. Il paraît préférable de s'engager dans une réflexion au niveau local en terme de stratégie d'aménagement agricole et rural dans le cadre, en particulier des SAGE. Si l'irrigation est utile pour accroître ou régulariser le revenu des agriculteurs, maintenir des capacités de concurrence, assurer une utilisation optimale de l'espace tout en ayant un impact sur l'environnement acceptable, dans un secteur bien défini, elle pourra continuer à se développer. En contrepartie les agriculteurs devront accepter de recourir à d'autres solutions quand l'irrigation ne pourra être justifiée.

\section{Annexe II}

Evolution des surfaces irriguées par type de cultures

\begin{tabular}{|l|r|r|r|}
\hline & 75 & 88 & $\begin{array}{c}\text { indice } \\
88 / 75\end{array}$ \\
\hline Maïs grain & 229000 & 575000 & 250 \\
Betteraves sucrières & 15500 & 26500 & 171 \\
Cultures permanentes & 98000 & 121000 & 123 \\
Vignes & 44000 & 12000 & 27 \\
Fourrages & 58000 & 94000 & 162 \\
S.T.H. & 71500 & 47000 & 66 \\
Pommes de terre & 13000 & 16000 & 123 \\
Légumes de plein champ & 39000 & $81000(79)$ & 207 \\
Oléoprotéagineux & & 85000 & \\
\hline
\end{tabular}

\section{Annexe III}

\section{Investissements d'irrigation * cumulés (1981-1988)} (en milliers de francs)

\begin{tabular}{|c|c|c|c|}
\hline & $\begin{array}{l}\text { Investis- } \\
\text { sements }\end{array}$ & Subvention & \\
\hline Total France métro. & 9006000 & 5091000 & $56,5 \%$ \\
\hline Régions peu concernées & 363800 & 199600 & $55 \%$ \\
\hline Régions les plus concernées & 8642200 & 4891400 & $56,6 \%$ \\
\hline Régions méditerranéennes & 4086700 & 2371200 & $58 \%$ \\
\hline Autres régions concernées & 4555500 & 2520200 & $55 \%$ \\
\hline
\end{tabular}

$\left(^{*}\right)$ Réseaux, retenues collinaires et grands barrages.

\section{Bibliographie}

[1] AGRESTE. - Etude $n^{\circ}$ 13, décembre 1991.

[2] Conseil Général du G.R.E.F. - Réflexions sur certains aspects techniques et économiques de l'irrigation en France (document interne).

\section{Annexe I}

1988 : Importance des superficies irriguées

\begin{tabular}{|l|r|r|r|}
\hline & $\begin{array}{l}\text { SUPERFICIES } \\
\text { IRRIGUEES }\end{array}$ & S.U.U. & \multicolumn{1}{l|}{$\%$} \\
\hline METROPOLE & 1146910 & 28662630 & $4,0 \%$ \\
\hline & & & \\
\hline NORD PAS DE CALAIS & 2399 & 877628 & $0,3 \%$ \\
\hline PICARDIE & 12739 & 1355904 & $0,9 \%$ \\
\hline ILE DE FRANCE & 12503 & 595040 & $2,1 \%$ \\
\hline CENTRE & 149537 & 2450018 & $6,1 \%$ \\
\hline HAUTE NORMANDIE & 2757 & 819741 & $0,3 \%$ \\
\hline & & & \\
\hline BASSE NORMANDIE & 4072 & 1356654 & $0,3 \%$ \\
\hline BRETAGNE & 6809 & 1757126 & $0,4 \%$ \\
\hline PAYS-DE-LA-LOIRE & 76171 & 2309109 & $3,3 \%$ \\
\hline POITOU-CHARENTES & 98510 & 1788062 & $5,5 \%$ \\
\hline LIMOUSIN & 2074 & 886722 & $0,2 \%$ \\
\hline & & & \\
\hline AQUITAINE & 229658 & 1542006 & $14,9 \%$ \\
\hline MIDI-PYRENEES & 209891 & 2397016 & $8,8 \%$ \\
\hline CHAMPAGNE-ARDENNE & 4797 & 1558724 & $0,3 \%$ \\
\hline LORRAINE & 587 & 1116009 & $0,1 \%$ \\
\hline ALSACE & 33537 & 329345 & $10,2 \%$ \\
\hline & & & \\
\hline FRANCHE COMTE & 722 & 682888 & $0,1 \%$ \\
\hline BOURGOGNE & 13648 & 1797469 & $0,8 \%$ \\
\hline AUVERGNE & 12662 & 1589055 & $0,8 \%$ \\
\hline RHONE-ALPES & 76480 & 1647479 & $4,6 \%$ \\
\hline LANGUEDOC- ROUSSILLON & 70785 & 1019836 & $6,9 \%$ \\
\hline & 116126 & 661541 & $17,6 \%$ \\
\hline PROV. ALP. COTE D'AZUR & 10446 & 125254 & $8,3 \%$ \\
\hline CORSE & & & \\
\hline & & & \\
\hline & & & \\
\hline & & & \\
\hline
\end{tabular}

Colonne 1 : superficie effectivement irrigute en ha (RGA 1988)

2 : superflic agricole utiliste des exploitations en ha (RGA 1988)

3 : part de la S.A.U. irriguée (en \%)

*

\section{Accroissement des superficies irriguées (1970-1988)}

\begin{tabular}{|l|r|r|r|}
\hline \multirow{2}{*}{ Région } & \multicolumn{2}{|c|}{$\begin{array}{c}\text { Surfaces irriguées } \\
\text { (en milliers d'ha) }\end{array}$} & $\begin{array}{c}\% \\
\text { d'augmen- } \\
\text { tation }\end{array}$ \\
\cline { 2 - 3 } & 1970 & 1988 & \\
\hline Centre & 66,8 & 149,5 & 124 \\
Pays de la Loire & 22,9 & 76,2 & 232 \\
Poitou-Charentes & 8,0 & 98,5 & 1130 \\
Aquitaine & 67,7 & 229,7 & 240 \\
Midi-Pyrénées & 60,5 & 210,0 & 247 \\
Alsace & 6,8 & 33,6 & 394 \\
Rhône-Alpes & 36,8 & 76,5 & 108 \\
\hline
\end{tabular}

Ministère de l'agriculture et de la forêt.

Direction de l'espace rural et de la forêt. 